\title{
Recommendation for Inclusion of Surface Echocardiography in Evaluation of Chest Pain in Acute Emergency Care
}

\author{
Viju Wilben ${ }^{1}$ Dhruvin Limbad ${ }^{2}$ Bijay BS ${ }^{3} \quad$ Srinath Kumar TS ${ }^{1} \quad$ Muralidhar Kanchi ${ }^{4}$
}

\footnotetext{
${ }^{1}$ Department of Cardiac Anaesthesia, Narayana Institute of Cardiac Sciences, Bangalore, India

2 Resident Emergency Department, Narayana Institute of Cardiac Sciences, Bangalore, India

${ }^{3}$ Department of Emergency Medicine, Mazumdar Narayana Institute of Cardiac Sciences, Bangalore, India

${ }^{4}$ Department of Anaesthesia, Narayana Institute of Cardiac

Sciences, Narayana Health City, Bangalore, India
}

J Card Crit Care 2021;5:97-102.

\begin{abstract}
Address for correspondence Muralidhar Kanchi, MD, FIACTA, FICA, MBA, FASE, PhD, Department of Anaesthesia and Intensive Care, Narayana Institute of Cardiac Sciences, Narayana Health City, Narayana Hrudayalaya Institute of Allied Health Sciences, 258/A, Bommasandra Industrial Area, Anekal Taluk, Bangalore-560 099, Karnataka, India (e-mail: muralidhar.kanchi.dr@narayanahealth.org, kanchirulestheworld@gmail.com).
\end{abstract}

\section{Abstract}

\section{Keywords}

- echocardiography

- chest pain

- acute coronary syndrome
Objective A significant number of conditions may mimic acute myocardial infarction when patients present to acute emergency care (AEC) with chest pain. A proportion of such patients may exhibit ST segment abnormality on the electrocardiogram (ECG) which is due to conditions other than acute coronary syndromes (ACS) or myocardial infarction. The American Heart Association/American College of Cardiology guidelines (2015) algorithm for ACS does not include echocardiographic evaluation in the assessment of chest pain. Patients with chest pain may be subjected to investigations and interventions based on ECG leading unwarranted invasive procedures, which may prove unnecessary, futile, and even detrimental. This study was performed to determine if a bedside echocardiography would help identify the conditions that do not need intervention and might possibly change the treatment pathway at the right time. Materials and Methods In a prospective observational study design, adult patients presenting to AEC with chest pain were included in the study. After the assessment of airway, breathing and circulation, and initiation of bed side monitoring, a 12-lead ECG was obtained. Patients exhibiting a significant ST change on ECG were subjected to bedside echocardiography, that is, two-dimensional (2D) transthoracic echocardiography (2D-TTE) with a cross reference to a consultant cardiologist for the precise assessment and diagnosis. The findings of echocardiography were correlated with electrocardiogram for possible diagnostic coronary angiography and percutaneous coronary intervention. The results of ECG, echocardiography, and coronary angiography (if done) were analyzed to determine the sensitivity and specificity of echocardiography for ACS.

Results Among 385 patients in the study, 312 were suspected to suffer acute coronary syndrome; among these patients, eight patients turned out to have chest pain due to non-ACS. Of the 73 patients, the chest pain was suspected to be not of cardiac ischemia origin; among these patients, 66 patients were true negative and 7 patients were false positive. Echocardiography was the predictive of ischemic chest pain with a predictive value of $97.7 \%$. The specificity of echocardiography calculated from the
DOI https://doi.org/

$10.1055 / \mathrm{s}-0041-1723930$ ISSN 2457-0206. (c) 2021. Official Publication of The Simulation Society (TSS), accredited by International Society of Cardiovascular Ultrasound (ISCU).

This is an open access article published by Thieme under the terms of the Creative Commons Attribution-NonDerivative-NonCommercial-License, permitting copying and reproduction so long as the original work is given appropriate credit. Contents may not be used for commercial purposes, or adapted, remixed, transformed or built upon. (https://creativecommons.org/licenses/by-nc-nd/4.0/)

Thieme Medical and Scientific Publishers Pvt. Ltd. A-12, 2nd Floor, Sector 2, Noida-201301 UP, India 
above confusion matrix was $90.4 \%$ and sensitivity was $97.4 \%$. The positive predictive value of 2D-TTE was $97.7 \%$ and negative predictive value was $89.1 \%$. The overall accuracy of bedside 2D-TTE was $96.1 \%$.

Conclusion Echocardiography was found to be an effective tool in aiding diagnosis of a patient presenting to AEC with chest pain and ST-T changes in ECG. A significant percentage of patients (18.7\%) presented to AEC with chest pain, ST-T changes and found to have causes other than ACS, and screening echocardiography (2D-TTE) was able to identify $90.4 \%$ of those cases. From this study, we conclude that bedside echocardiography had high specificity $(90.4 \%)$ and sensitivity $(97.43 \%)$ in identifying regional wall motion abnormality due to ACS. Hence, bedside echocardiography is recommended in patients with chest pain and ST-segment abnormality to avoid unnecessary delay in diagnosis and invasive interventions in non-ACS.

\section{Introduction}

"Time is muscle" is a phrase established for the need of rapid diagnosis and swift initiation of therapy in patients with ST elevation myocardial infarction (STEMI). Therapy includes thrombolytic therapy or percutaneous coronary intervention. The 2015 Advanced Cardiac Support (ACLS)/American Heart Association (AHA) algorithm for acute coronary syndrome does not include bedside echocardiography in the pathway of management of chest pain in the emergency. The time between the onset of symptoms and initiation of therapy is of paramount importance. Though these procedures are lifesaving, if done in patients who suffer from chest pain due to noncoronary artery disease related lesions, they may prove unnecessary adding to expenses, waste of resources, and lead to life threatening complications as well as. ${ }^{1-4}$ The ST segment is the flat, iso-electric section of the ECG which represents the interval between ventricular depolarization and repolarization. ${ }^{5}$ Injury currents flowing from the depolarized ischemic regions to normal regions result in the appearance of ST segment elevation where the ischemic region is transmural. ${ }^{5}$ Conditions mimicking ST segment elevation have been reported in several published reports. It was found that a proportion of patients with suspected ST segment changes suffer from other cardiac conditions such as aortic stenosis, pericarditis, left ventricular hypertrophy, Brugada syndrome, etc. Additionally, noncardiac conditions like aortic dissection, subarachnoid hemorrhage, pneumonia, peritonitis, etc. may also cause ST changes on the ECG. ${ }^{6-9}$ Abdominal condition like acute pancreatitis and acute cholecystitis can also mimic ST segment changes which are of noncardiac origin. ${ }^{10}$

\section{Materials and Methods}

In a cross-sectional observational study conducted in emergency department at a tertiary care cardiac hospital between November 2017 and October 2019, all adult patients of age 18 years or greater, presenting with chest pain for the first time and ST segment alterations in the 12 lead ECG were included for analysis. Patients with known history of cardiac disease, abdominal conditions, and patient's status postpercutaneous transluminal angioplasty/coronary artery bypass grafting were excluded from the study. The study was conducted after obtaining ethical committee approval. The ACLS guidelines proposed by AHA and American College of Cardiology were initiated. In brief, once the patient presented to the emergency department with chest pain after obtaining a targeted history, clinical examination, and initial assessment of the patient, oxygen was administered if the patient was obviously dyspneic, had obvious signs of heart failure, and/or had an initial oxygen saturation of less than $94 \%{ }^{11}$ If symptoms and signs were suggestive of myocardial ischemia or infarction, $150 \mathrm{mg}$ of nonenteric aspirin was administered in a chewable form to hasten absorption. ${ }^{12-15}$ Additionally, the subjects were given up to three doses of nitroglycerine (tablets or spray) at intervals of 3 to 5 minutes. Nitrates were not administered in patients with initial systolic blood pressure $<90$ or $\geq 30 \mathrm{mmHg}$ below baseline and in patients with right ventricular (RV) infarction, ${ }^{16-18}$ in patients with known inferior wall STEMI, and suspected RV involvement and when patients have taken a phosphodiesterase- 5 inhibitor within 24 hours (48 hours for tadalafil). ${ }^{19}$

A 12-lead ECG was obtained along with initial assessment after establishing the bedside monitoring. If a significant ST change is observed in ECG, the patient was subjected to a bedside echocardiogram with a cross reference to the cardiologist about further protocols for possible reperfusion therapy. Two-dimensional transthoracic echocardiography (2D-TTE) was performed by using the Philips CX-50 by an experienced sonologist, who was blinded to the patient's status. A phased array transducer with a frequency range of 1 to $5 \mathrm{MHz}$ was used to perform echocardiographic examination. ${ }^{20}$ Standard 2D-TTE examination was used that included (1) apical four chamber and two chamber views, (2) para-sternal short and long axis, (3) subcostal views for cardiac chambers and inferior venacava, (4) thoracic aorta and upper abdominal aorta, (5) pleural views, and (6) lung scan. Color Doppler imaging was performed for the evaluation of intracardiac shunting and valvular regurgitation. Motion mode (M-mode) was 
used where appropriate. Results of 2D-TTE were recorded for analysis. The findings of 2D-TTE were correlated with electrocardiogram and diagnostic coronary angiography, if it is done, to determine the sensitivity and specificity of echocardiography. Data of patients age, sex, history of smoking, comorbidity such as diabetes, presenting complaints, prehospitalization treatment, duration between complaint and hospital admission, electrocardiogram, cardiac enzyme study, echocardiography findings, final outcome, ICU stay duration, requirement of ventilatory support, and complications were acquired ( - Fig. $\mathbf{1}$ ).

\section{Sample Size}

Based on a previous study involving 80 patients, ${ }^{21}$ in 65 patients echocardiography was adequately performed, with 33 patients having evidence of clinical MI and 32 patients (49\%) without the evidence of clinical MI, with confidence

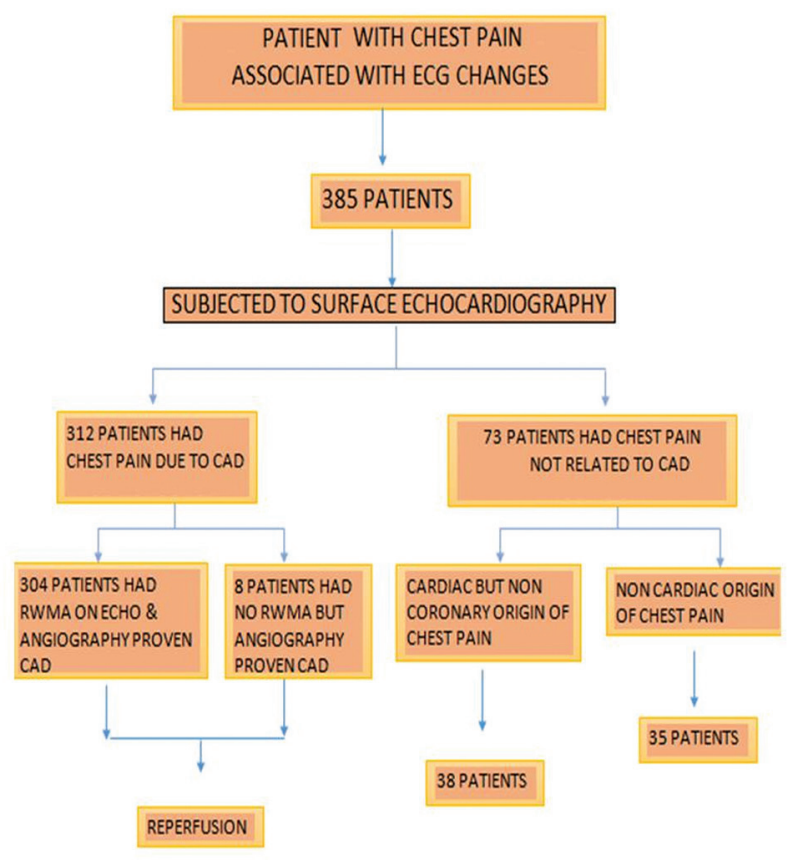

Fig. 1 Flowchart for patient management. interval $(\mathrm{CI})=95 \%$, and precision $(\mathrm{d})=5 \%$, the calculated sample size was 384. Statistical analysis was performed by the software SPSS 22.0 version. Continuous variables will be was expressed as mean \pm standard deviation and categorical variables as frequencies or percentages. Specificity and sensitivity of echocardiography in detecting regional wall motion abnormality was determined.

- True positive: ACS (angiography) + RWMA (TTE)

- True negative: non-ACS (angiography) + no RWMA (TTE)

- False positive: non-ACS (angiography) + RWMA (TTE)

- False negative: ACS (angiography) + no RWMA (TTE)

\section{Results}

There were a total of 385 patients who presented to the emergency with acute chest pain for the first time during the study period. The mean age of the study population was 55.08 \pm 14.17 years (-Table1). Among these, 142 patients were from the age group over 60 years (36.88\%), closely followed by 104 subjects in the age group 51 to 60 years (27\%). There were significantly less number of patients, that is, five subjects $(1.3 \%)$ in the study group who were less than 20 years. Among the 385 study subjects, 312 patients (81.03\%) had final diagnosis as acute coronary syndrome (ACS) while 73 patients (18.9\%) had an alternative diagnosis (-Table 1). Majority of the patients with diagnosis as ACS had STEMI, 197 patients (63.14\%) while 113 patients (36.22\%) had NSTEMI as their diagnosis (-Table 2). There were 18 alternative diagnosis to patients presenting to ER with chest pain and ECG changes in our study. Most patients in the non-ACS group had final diagnosis as pulmonary embolism with 13 patients (17.8\%) closely followed by pancreatitis with nine patients (12.3\%). Hypertrophic cardiomyopathy (HOCM) and aortic syndromes each had 8 patients (10.9\%) (-Table 3 ). Among the 385 patients in the study, 304 patients were true positive, 66 patients were true negative, 7 patients were false positive, and 8 patients were false negative. The specificity of echocardiography calculated from the above confusion matrix was $90.4 \%$ and sensitivity was $97.4 \%$. The positive predictive value of ECHO was $97.7 \%$ and negative predictive value was $89.1 \%$. The overall accuracy of bedside echocardiography was $96.1 \%$.

Table1 Demographic and clinical data

\begin{tabular}{|l|l|l|l|}
\hline \multicolumn{4}{|c|}{ Demographic data } \\
\hline \multirow{4}{*}{ Age } & $\leq 20$ & $5(1.3 \%)$ & $(3.9 \%)$ \\
\cline { 2 - 4 } & $21-30$ & 15 & $(9.87 \%)$ \\
\cline { 2 - 4 } & $31-40$ & 38 & $(21.04 \%)$ \\
\cline { 2 - 4 } & $41-50$ & 81 & $(27.01 \%)$ \\
\cline { 2 - 4 } & $51-60$ & 104 & $(36.88 \%)$ \\
\cline { 2 - 4 } & $\geq 61$ & 142 & $(81.03 \%) / 73(18.96 \%)$ \\
\hline Siagnosis & ACS/non-ACS & 312 & $(81.3 \%) / 72(18.7 \%)$ \\
\hline
\end{tabular}

Abbreviation: ACS, acute coronary syndrome.

Note: $n=385$ (percentage parenthesis). 
Table 2 Distribution of diagnosis among the acute coronary syndrome group of patients

\begin{tabular}{|l|l|l|l|}
\hline \multicolumn{4}{|c|}{ Clinical data of ACS group } \\
\hline STEMI/NSTEMI/vasospastic angina & 197 & $(63.14 \%) / 113(36.22 \%) / 2(0.64 \%)$ \\
\hline ICU/HDU stay & 274 & $(87.82 \%) / 38$ & $(12.18 \%)$ \\
\hline Oxygen requirement: yes/no & 107 & $(34.3 \%) / 205$ & $(65.7 \%)$ \\
\hline Mechanical ventilatory support: yes/no & $36(11.55 \%) / 276$ & & $(84.46 \%)$ \\
\hline Outcome: discharge/mortality & 294 & $(94.23 \%) / 18$ & $(5.76 \%)$ \\
\hline
\end{tabular}

Abbreviations: ACS, acute coronary syndrome; HDU, high dependency unit; ICU, intensive care unit; NSTEMI, non-ST elevation myocardial infarction; STEMI, ST-elevation myocardial infarction.

Note: $n=312$ (percentage parenthesis).

Table 3 Distribution of diagnosis among the nonacute coronary syndrome group

\begin{tabular}{|l|l|l|}
\hline Pulmonary embolism & \multicolumn{2}{|c|}{$13(17.81 \%)$} \\
\hline Acute pancreatitis & 9 & $(12.3 \%)$ \\
\hline HOCM & 8 & $(11 \%)$ \\
\hline Aortic syndromes & 8 & $(11 \%)$ \\
\hline Acute cholecystitis & 7 & $(9.59 \%)$ \\
\hline Pneumothorax & 7 & $(9.59 \%)$ \\
\hline Myocarditis & 5 & $(6.85 \%)$ \\
\hline Pneumonia & 3 & $(4.11 \%)$ \\
\hline SAH & 3 & $(4.11 \%)$ \\
\hline Hypokalemia & 2 & $(2.74 \%)$ \\
\hline Acute appendicitis & 1 & $(1.37 \%)$ \\
\hline Cardiac contusion & 1 & $(1.37 \%)$ \\
\hline Digoxin toxicity & 1 & $(1.37 \%)$ \\
\hline Empyema & 1 & $(1.37 \%)$ \\
\hline Liver abscess & 1 & $(1.37 \%)$ \\
\hline Mitral valve prolapse & 1 & $(1.37 \%)$ \\
\hline Pericardial effusion & 1 & $(1.37 \%)$ \\
\hline Pulmonary aspergillosis & 1 & $(1.37 \%)$ \\
\hline
\end{tabular}

Abbreviations: HOCM, hypertrophic obstructive cardiomyopathy; SAH, subarachnoid hemorrhage.

The final outcome of death or discharge was 6 and 94\%, respectively. Of the patients who were admitted to hospital, $88 \%$ patients required ICU stay. Average hospital stay was $4 \pm 3.5$ days while average ICU stay was $2 \pm 2.75$ days. Out of total admitted patients, $34 \%$ patients required oxygen to maintain saturation of more than $94 \%$, while $12 \%$ patients required mechanical ventilation either noninvasive or invasive or respiratory distress. Fifty-four patients, who were referred to this institution from other health care facilities, were subjected to treatment at the initial primary hospital that included dual anti platelets administration (34 patients), medical thrombolysis (17 patients), and endotracheal intubation and mechanical ventilation ( 3 patients). Pulmonary complications including pulmonary edema and respiratory tract infection occurred in 15 patients. Acute kidney injury requiring renal replacement therapy was needed in 22 patients. Cardiogenic shock and cardiac arrest occurred in 25 patients while various types of conduction block developed in 19 patients. Supraventricular tachyarrhythmia developed in 12 patients. Cerebral stroke developed in three patients while diabetic ketoacidosis developed in three patients. Five patients in the cohort admitted to ICU received intra-aortic balloon pump for as a temporary mechanical support of the failing heart. One patient developed hematemesis while one patient developed cardiac tamponade.

\section{Discussion}

Accurate and precise diagnosis of patients presenting to the AEC with chest pain is absolutely essential for successful outcome. 2D-TTE is a diagnostic and monitoring tool which is readily available in most emergency care departments worldwide. 2D-TTE can be done rapidly as a point-of-care procedure that provides very valuable information of the hemodynamic status, cardiovascular system, pleura, and the lungs. 2D-TTE is helpful in efficiently delineating patients with aortic stenosis, acute aortic dissection, and avoids discharging patients with AMI. On the other hand, 2D-TTE may help in triaging patients such that the patient ICU beds are correctly allocated to deserving patients such as those with myocardial ischemia/infarction. This will avoid patients occupying expensive, intensive care beds, and substantially increasing the financial cost of diagnosis. In a study published, the influence of real-time 2D-TTE on medical decision making in the ED was analyzed in 100 consecutive patients presenting with suspected cardiac pathology. ${ }^{21}$ Echocardiography altered the diagnosis in $37 \%$ of patients, led to changes in treatment in $25 \%$ of patients, and increased physician confidence in their diagnosis in $50 \%$ of cases $^{21}$. In addition, the latest information available on the use of early 2D-TTE the prediction of cardiac events in AEC in patients with chest pain was as follows: sensitivity of $91 \%$ (95\% confidence interval [CI]: 79-97\%), which was higher than that of ECG (40\%, 95\% CI: $27-55 \%$; $p<0.0001){ }^{22}$ Specificity of echocardiography was $75 \%$ (95\% CI: 69-81\%), which was significantly lower than that of ECG (94\%, 95\% CI: 90-97\%; $p<0.0001$ ). Positive predictive values were not significantly different (44\%, 95\% CI: $34-54 \%$ and $60 \%, 95 \% \mathrm{CI}: 42-76 \% ; p=0.12) .{ }^{22}$

In the present study, among 385 patients who presented with chest pain, 312 patients (81\%) had ACS while 73 patients (18\%) had diagnoses other than ACS. In the non-ACS limb of the study most common diagnosis was pulmonary embolism (17.81\%), followed by acute pancreatitis (12.3\%) and HOCM (10.9\%) and aortic syndromes (10.9\%). In the ACS hand of the study, the most common diagnosis was STEMI (62.5\%) followed by NSTEMI (36.22\%). In a study conducted in 2006 to 
detect the prevalence of normal coronary angiography in patients, presenting with ST changes in ECG, noticed that 26\% were misdiagnosis. In contrast to our study, they have noticed that the most common misdiagnosis was myocarditis (19\%), but it is closely followed by pulmonary embolism (12\%). ${ }^{14}$

Bedside echocardiography was used to identify regional wall motion abnormality in patients presenting to emergency department with chest pain and ECG changes suggestive of ACS.

Coronary angiography was considered to be the gold standard for diagnosing ACS. In our study, we noticed that 304 patients were true positive and 66 patients were true negative cases. Bedside screening echocardiography had a specificity of $90.4 \%$ and sensitivity of $97.4 \%$. Positive predictive value of screening echocardiography in diagnosing ACS was $97.7 \%$ and negative predictive value was $89.1 \%$. It was found that echo has an accuracy of $96.1 \%$ in identifying
ACS in patients with chest pain. In a study about immediate diagnosis of MI by ECHO, it was noticed that ECHO identified RWMA in 31 (94\%) of the 33 ACS patients, that is, true positives and of the 32 patients who did not have ACS, screening echocardiography did not show any RWMA in 27 of the patients (84\%), that is, true negative. It was concluded that ECHO has a sensitivity of $93.9 \%$ and specificity of $84.38 \%$ in detecting ACS. ${ }^{23}$

\section{Conclusion}

A considerable number of patients (18.7\% in our study) presented to AEC with chest pain associated with ECG changes that were found to have noncoronary origin of chest pain. Echocardiography is feasible and effective in aiding diagnosis of a patient presenting with chest pain and ECG changes. Echocardiography had high sensitivity (97.4\%) and specificity

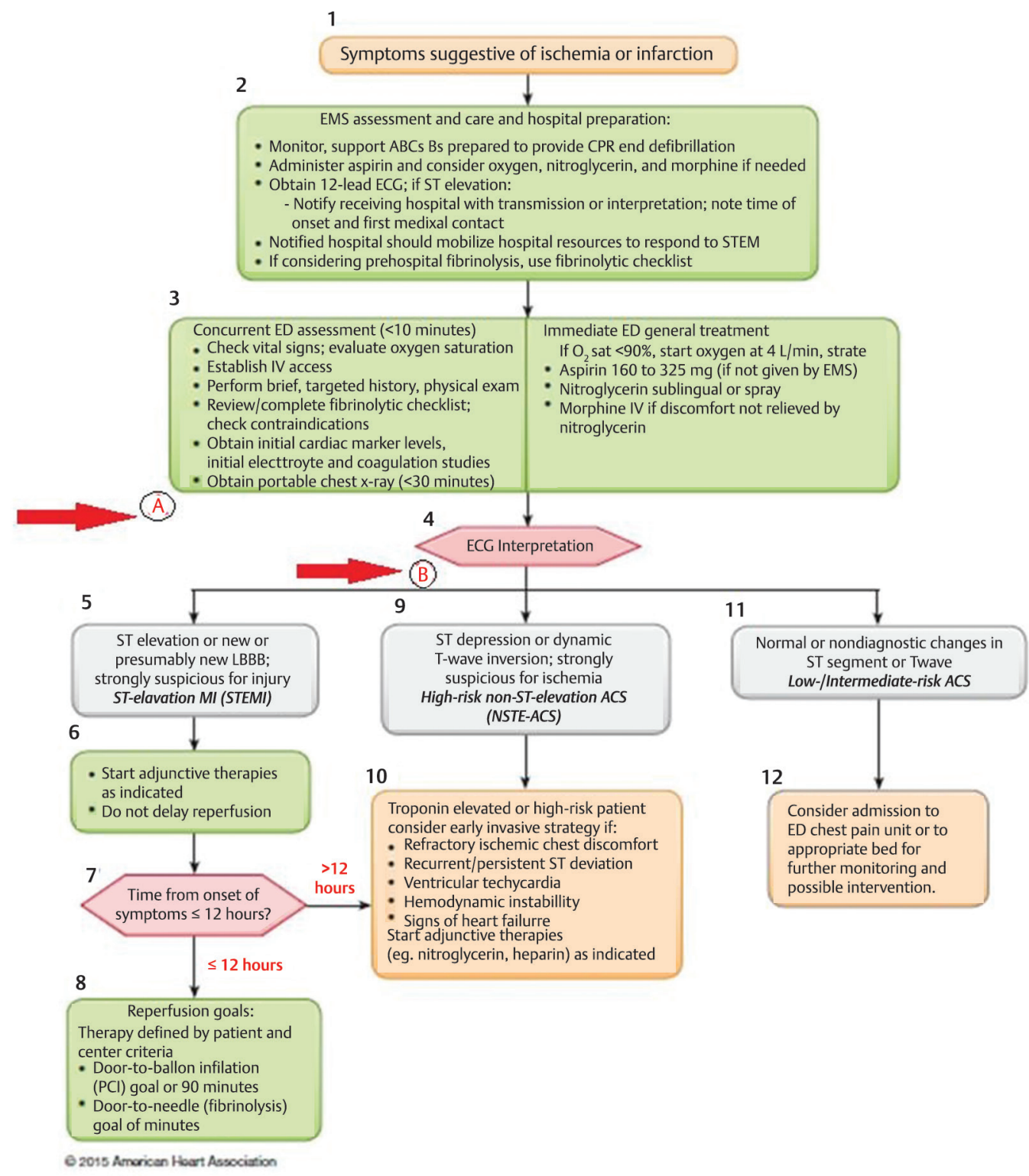

Fig. 2 Acute coronary syndromes algorithm 2015 update. Arrows indicate the time points of bed-side echocardiography. 
(90.4\%) in identifying RWMA; hence, screening echocardiography might help to avoid delay in diagnosis and other invasive intervention in non-ACS patients.

\section{Note}

We recommend the inclusion of bedside echocardiography in the algorithm for the evaluation of patients with chest pain and ECG changes in the AEC ( - Fig. 2). The basic format of the AHA 2015 algorithm is maintained. The surface echocardiography can be included either at point $A$ or $B$ (shown in the red arrow).

\section{Funding}

None.

\section{Conflict of Interest}

None declared.

\section{References}

1 Peter F, Ludman. National Audit of Percutaneous Coronary Interventions Annual Report 2015. Birmingham:; 2016

2 Dobies DR, Barber KR, Cohoon AL. Validity of a PCI Bleeding Risk Score in patient subsets stratified for body mass index. Open Heart 2015;2(1):e000088

3 Tavakol M, Ashraf S, Brener SJ, Risks and complications of coronary angiography: a comprehensive review. Am Global J Health Sci2012;;4(1)

4 George S, Butler R, Nolan J, Mamas MA. Percutaneous coronary intervention and bleeding complications. Eur Med J 2016

5 Rautaharju PM, Surawicz B, Gettes LS, et al; American Heart Association Electrocardiography and Arrhythmias Committee, Council on Clinical Cardiology; American College of Cardiology Foundation; Heart Rhythm Society; Endorsed by the International Society for Computerized Electrocardiology. AHA/ACCF/HRS recommendations for the standardization and interpretation of the electrocardiogram: part IV: the ST segment, $\mathrm{T}$ and $\mathrm{U}$ waves, and the QT interval. J Am Coll Cardiol 2009;53(11):982-991

6 Edhouse J, Brady WJ, Morris F. ABC of clinical electrocardiography: acute myocardial infarction-part II. BMJ 2002;324(7343) :963-966

7 Spittell PC, Spittell JA Jr, Joyce JW, et al. Clinical features and differential diagnosis of aortic dissection: experience with 236 cases (1980 through 1990) Mayo Clin Proc 1993;68(7):642-651

8 Ryan ET, Pak PH, DeSanctis RW. Myocardial infarction mimicked by acute cholecystitis. Ann Intern Med 1992;116(3):218-220
9 Fulton MC, Marriott HJ. Acute pancreatitis simulating myocardial infarction in the electrocardiogram. Ann Intern Med 1963;59:730-732

10 Antonelli D, Rozner E, Turgeman Y. Unusual electrocardiographic changes during acute pancreatitis. Cor Vasa 2017;59(5):e446-e449

11 Wijesinghe $M$, Perrin $K$, Ranchord A, Simmonds $M$, Weatherall M, Beasley R. Routine use of oxygen in the treatment of myocardial infarction: systematic review. Heart 2009;95(3):198-202

12 Freimark D, Matetzky S, Leor J, et al. Timing of aspirin administration as a determinant of survival of patients with acute myocardial infarction treated with thrombolysis. Am J Cardiol 2002;89(4):381-385

13 Haynes BE, Pritting J. A rural emergency medical technician with selected advanced skills. Prehosp Emerg Care 1999;3(4):343-346

14 Funk D, Groat C, Verdile VP. Education of paramedics regarding aspirin use. Prehosp Emerg Care 2000;4(1):62-64

15 Verheugt FW, van der Laarse A, Funke-Küpper AJ, Sterkman LG, Galema TW, Roos JP. Effects of early intervention with low-dose aspirin (100 mg) on infarct size, reinfarction and mortality in anterior wall acute myocardial infarction. Am J Cardiol 1990;66(3):267-270

16 Bussmann WD, Passek D, Seidel W, Kaltenbach M. Reduction of CK and CK-MB indexes of infarct size by intravenous nitroglycerin. Circulation 1981;63(3):615-622

17 Charvat J, Kuruvilla T, al Amad H. Beneficial effect of intravenous nitroglycerin in patients with non-Q myocardial infarction. Cardiologia 1990;35(1):49-54

18 Jugdutt $\mathrm{BI}$, Warnica JW. Intravenous nitroglycerin therapy to limit myocardial infarct size, expansion, and complications. Effect of timing, dosage, and infarct location. Circulation 1988;78(4):906-919

19 Madsen JK, Chevalier B, Darius H, et al. Ischaemic events and bleeding in patients undergoing percutaneous coronary intervention with concomitant bivalirudin treatment. EuroIntervention 2008;3(5):610-616

20 Levitt MA, Jan BA. The effect of real time 2-D-echocardiography on medical decision-making in the emergency department. J Emerg Med 2002;22(3):229-233

21 Horowitz RS, Morganroth J, Parrotto C, Chen CC, Soffer $\mathrm{J}$, Pauletto FJ. Immediate diagnosis of acute myocardial infarction by two-dimensional echocardiography. Circulation 1982;65(2):323-329

22 Kontos MC, Arrowood JA, Paulsen WH, Nixon JV. Early echocardiography can predict cardiac events in emergency department patients with chest pain. Ann Emerg Med 1998;31(5):550-557

23 Anjali VS, Page S. Echocardiographic assessment of left ventricular function in patients of acute myocardial infarction. Int J Adv Med 2017;4:926-931 